\title{
HACIA UNA TEORÍA SISTÉMICO-CIBERNÉTICA DEL DERECHO
}

\author{
Ernesto GRÜN \\ Andrés BOTERO BERNAL
}

Resumen:

En el presente texto se presentará al lector cómo la sistémica y la cibernética sirven al derecho para estructurar así una Teoría Sistémico-Cibernética del Derecho (TSCD). Mediante ésta se podrá dar respuesta a diversos interrogantes iusfilosóficos, obteniendo una visión del derecho más completa de las que hasta ahora se nos ha ofrecido. Para lograr tal cometido, se expondrá la manera cómo la metodología y la epistemología sistémica y cibernética irrumpen en el mundo jurídico, para luego enumerar algunas características de una posible TSCD y los problemas a los que esta visión del derecho ofrece algo nuevo.

La TSCD constituirá - así lo esperamos- el paradigma que la complejidad de los temas y sistemas del derecho (nacionales, internacionales, trasnacionales, etcétera) requiere para hacer frente a la crisis de su funcionamiento práctico y de su investigación científica y filosófica.

Abstract:

This article will present how Systemics and Cybernetics might prove useful for law in advancing a Systemic-Cybernetic Theory of Law (TSCD). With TSCD it will be possible for legal scholars to solve several jurisprudential problems and also elaborate a new and more comprehensive approach to law than the ones currently available. In order to achieve this goal, it will be argued how systemic-cybernetic methodology and epistemology have come into play in the law, and which kind of features are included in the theory, and also it will be added where new answers are offered.

The authors hope TSCD to be considered as a paradigm helpful for facing the challenges and crisis that in their practical operation and philosophical and scientific research are being put forward by different systems of law (national, international, transnational, etcetera). 
SUMARIO: I. Introducción. II. Teorías de los sistemas jurídicos. III. Características de la TSCD. IV. Conclusiones. V. Bibliografía.

\section{INTRODUCCIÓN}

Hemos creído y considerado que tanto la sistémica como la cibernética constituyen útiles herramientas epistemológicas y metodológicas de lectura de lo jurídico y por tanto elementos fundamentales para la construcción de un nuevo modelo iusfilosófico. ${ }^{1}$ Mediante ellas, estimamos, pueden resolverse diversas dudas iusfilosóficas y obtenerse una más visión más apropiada del derecho en el siglo XXI. A las respuestas que resulten de la aplicación de dicha metodología y epistemología en los debates iusfilosóficos y a ese cuadro más dinámico y complejo, es a lo que llamariamos una teoría sistémico-cibernética del derecho (en adelante TSCD).

Mediante el presente artículo pretendemos efectuar una primera aproximación a ella, a través de una reelaboración y sintesis de lo que han expuesto sobre el tema sus autores tanto en trabajos impresos como en publicaciones efectuadas en Internet, ${ }^{2}$ con el fin de ir elaborando una teoría del

1 Para que el lector pueda formarse una idea de las nociones básicas de sistémica y cibernética puede verse: Grün, Ernesto, "El derecho en el mundo globalizado del siglo XXI desde una perspectiva sistémico-cibernética” (en línea), Revista Telemática de Filosofía del Derecho, núm. 4 (2000/2001), capítulos I y II, http:// www.filosofiayderecho.com/rtfd/numero4/globalizado.htm; Grün, Ernesto, Una visión sistémica y cibernética del derecho en el mundo globalizado del siglo XXI, México-Buenos Aires, Lexis Nexis-UNAM, Instituto de Investigaciones Jurídicas, 2006. Grün, Ernesto, "La aplicación de la sistémica y la cibernética al derecho" (en línea), Panóptica, 2007; www.panoptica.org/8marcoabrilo7.htm.

2 Botero Bernal, Andrés, Diagnóstico de la eficacia del derecho en Colombia y otros ensayos, Medellín, Señal Editora y Fondo Editorial Biogénesis, 2003. Botero Bernal, Andrés, "Nuevos paradigmas científicos y su incidencia en la investigación jurídica”, Diálogos de Saberes, Bogotá, Centro de Investigaciones Socio Jurídicas, Facultad de Derecho, Universidad Libre, núm. 18-19, enero-diciembre de 2003, pp. 147-174. Grün, Ernesto, "Un nuevo enfoque para la teoría general del derecho", Revista La Ley, 1988, A, p. 979. Grün, Ernesto, "La teoría general de sistemas y la tributación", Revista La Información, t. LX, p. 1006. Grün, Ernesto, "Sistema jurídico y sistema ecológico. Un enfoque sistémico”, La Ley-Revista Actualidad, 19 
derecho más acorde con estos tiempos, tan diferentes de aquéllos de los positivistas y aún de los trialistas o tridimensionalistas y ni hablar de los de los jusnaturalistas, sean éstos de impronta teológica, racionalista, neoclásica, etcétera.

Ahora bien, ¿cómo iniciar nuestro acercamiento a una TSCD? Creemos que la manera de lograrlo es por medio de una reflexión sobre lo que en el mundo iusfilosófico se ha conocido como la teoría de los sistemas jurídicos. Esa será nuestra puerta de entrada a una TSCD, para luego centrarnos en la enunciación de sus características centrales que, como lo verá el lector, no son acabadas sino propuestas que se plantean. En fin, todo está por construir. Lo que aquí pretendemos es aportar ladrillos para ese edificio.

de agosto de 1993. Grün, Ernesto, "Un enfoque sistémico-cibernético de la mediación”, La Ley - Suplemento de Métodos Alternativos de Resolución de Conflictos, núm. 2. Grün, Ernesto, Una visión sistémica y cibernética del derecho, Buenos Aires, Abeledo Perrot, 1995. Grün, Ernesto, "La aplicación de la sistémica y la cibernética al derecho", en Botero Bernal, Andrés y Estrada Vélez, Sergio Iván (comp.), Temas de filosofía del derecho, Medellín, Señal editora y Universidad de Medellín, 2003. Grün, Una visión..., cit., nota 1, 2006, Grün, Ernesto, "Mediación: un enfoque sistémico-cibernético" (en línea), Transformación de Conflictos en el Mundo Hispánico; http://www.inter-mediacion.com/cibernetico.htm. Grün, Ernesto, "La globalización jurídica" (en línea), Transformación de conflictos en el Mundo Hispánico, http://www.inter-mediacion.com/grun.htm. Grün, Ernesto, "El derecho posmoderno: un sistema lejos del equilibrio" (en línea), Revista Telemática de Filosofía Del Derecho (Rtfd), núm. 1- 97/98, www.filosofiayderecho.com/rtfd/numero1/posmoder no.html. Grün, Ernesto, "La globalización del derecho: un fenómeno sistémico y cibernético" (en línea), Revista Telemática De Filosofía Del Derecho (Rtfd), núm. 2 (1998/1999), www.filosofiayderecho.com/rtfd/numero2/global.html. Grün, Ernesto, "Una visión sistémica y cibernética del derecho" (libro en versión electrónica), Revista Telemática De Filosofía Del Derecho (Rtfd), núm. 3 (1999/2000), www.filoso fiayderecho.com/rtfd/numero3/grun.htm. Grün, Ernesto, "Derecho y caos: sobre la actual y futura evolución del derecho" (en línea), Revista Telemática De Filosofía Del Derecho (Rtfd), núm. 3- 99/00, www.filosofiayderecho.com/rtfd/nume ro3/caos. $\mathrm{htm}$. Grün, Ernesto, "Los nuevos sistemas jurídicos del mundo globalizado" (en línea), Revista Telemática de Filosofía del Derecho (Rtfd), núm. 4 (2000/2001), www.filosofiayderecho.com/rtfd/numero4/istemas_ficha.htm. Grün, El derecho..., cit., nota 1, 2000/2001. Grün, Ernesto, "El derecho después del 11 de septiembre del 2001" (en línea), Revista Telemática de Filosofía del Derecho (Rtfd), núm. 5 (2001/2002), www.filosofiayderecho.com/rtfd/numero5/11-9-01.htm. Grün, Ernesto, "La crisis del sistema jurídico-político argentino. Un enfoque sistémico y cibernético" (en línea), Asociación Argentina de Filosofía del Derecho, Conferencias y Ponencias, www.aafd.org.ar/filosofia/documentos/16_Grun\%20Ernes to.doc. 
Para finalizar, agradecemos al profesor Enrique Cáceres Nieto por sus observaciones al presente escrito. Obviamente, la responsabilidad de lo dicho recae exclusivamente en nosotros.

\section{TEORÍAS DE LOS SISTEMAS JURÍDICOS}

Mucho se ha debatido en torno a si el derecho es sistema o no lo es. Y peor aún, en caso de aceptar la teoría de los sistemas jurídicos, qué entender por sistema. Es fácil observar que ha predominado en la teoría del derecho la concepción que lo identifica con un sistema, pero al momento de considerar qué entienden estas escuelas y autores por sistema encontramos una proliferación de significados.

Pero antes de entrar en ello queremos explicitar, muy brevemente, lo que es para nosotros un sistema (lo que no se corresponde con otras posturas que en la iusfilosofia se han dado y que más adelante se enunciarán), para lo cual usaremos las palabras de Charles François:

“(el sistema es) una entidad autónoma dotada de una cierta permanencia y constituida por elementos interrelacionados que forman subsistemas estructurales y funcionales, que se transforma dentro de ciertos límites de estabilidad gracias a regulaciones internas que le permiten adaptarse a las variaciones de su entorno específico"3 (p. ej. un hombre, un aparato de aire acondicionado, un automóvil, una ameba).

Un sistema, desde esta óptica, es un todo que funciona y que no puede ser dividido en partes independientes que cumplan esa misma función. Entonces, la condición de sistema no es una cualidad intrínseca de las cosas, sino una actitud o apreciación de cada uno, por lo que cuando decimos que tal cosa concreta o abstracta es un sistema deberíamos decir que la consideramos un sistema. En consecuencia, la sistémica no estudia a los sistemas a partir de

3 Véase François Charles, Diccionario de teoría de sistemas y cibernética, Buenos Aires, Gesi, 1991. p. 164. François, Charles (ed.), International Encyclopedia of Systemics and Cibernetics, 2a. ed., München, K.G.Knaur, 2004, t. II. p. 580. 
sus elementos básicos o últimos sino tratándolos a partir de su organización interna, sus interrelaciones recíprocas, sus niveles jerárquicos, su capacidad de variación y adaptación, su conservación de identidad, su autonomía, las relaciones entre sus elementos, sus reglas de organización y crecimiento, su desorganización y destrucción, etcétera.

Sin embargo, la forma como empezamos este capítulo pone en evidencia una tensión: vamos a hablar de sistema desde la tradición jurídica cuando para nosotros el concepto del que partimos nos arroja otro tipo de respuestas. Creemos, no obstante, que esta tensión se resolverá justo asumiendo la tradición en vez de desecharla, la cual en tres fases ha significado el concepto de sistema, queriendo nosotros afirmar que ahora emerge una cuarta significación del término, que es justo el concepto al que nos adscribimos.

Las diferentes concepciones tradicionales de sistema jurídico, al sentir de Norberto Bobbio, pueden ser clasificadas en tres grandes grupos. ${ }^{4}$ La primera acepción sería entender sistema como un orden derivado de una estructura jerárquica deductiva (como sería decir que el derecho es una expresión derivada de unos "principios generales del derecho" que irradian normas de inferior jerarquía hasta llegar a la norma concreta y particular en cascada normativa). De esta manera, existiría sistema en tanto hay normas superiores que dan unidad y criterio claro de identificación de lo que hace parte del mundo jurídico.

El segundo grupo considera sistema como un orden inductivo, algo así como lo que pregonó el realismo (tanto el sociológico de Santi Romano ${ }^{5}$ y de Olivecrona, ${ }^{6}$ como el judicial de $\operatorname{Ross}^{7}$ ), donde la jerarquía se establece de pautas

4 Bobbio, Norberto, Teoría general del derecho, Bogotá, Temis, 1987, pp. 181-183.

5 Romano, Santi, L'ordinamento giuridico, Firenze, Sansoni, 1946.

6 Olivecrona, Karl, El derecho como hecho, trad. J. J. Santa Piner, Buenos Aires, Depalma, 1959.

7 Ross, Alf, Sobre el derecho y la justicia, trad. Genaro Carrió, Buenos Aires, Eudeba, 1963. 
concretas en la sociedad que van en ascenso, permitiendo criterios de identificación de lo que hace parte de ese sistema, criterios que van de abajo hacia arriba. Claro está que Bobbio señala que el mejor ejemplo de este orden inductivo sería Savigny. Según Bobbio, cuando el alemán pregona la ciencia del derecho entiende el derecho como sistema en tanto pasa a ser ciencia (y como la ciencia es natural, entonces el sistema que tenía en mente Savigny era inductivo).

El tercer concepto, según Bobbio, es el siguiente: "Se dice que un ordenamiento jurídico constituye un sistema porque en él no pueden coexistir normas incompatibles". ${ }^{8}$ Así, el concepto de sistema del que parte Bobbio y toma en alguna medida de Kelsen es el de entender por sistema un edificio jurídico jerárquico y por tal lógico (en cuanto no hay contradicciones ni lagunas). 9 No es propiamente deductivo ni inductivo en la medida que no centra su argumento en que la identificación de lo que es derecho no viene tanto de prácticas sociales (la previsibilidad de la decisión judicial o la existencia de un conjunto normativo en un organismo social concreto, según el caso) sino de la imposibilidad de encontrar contradicciones y lagunas en la respuesta que el derecho da a quien le indaga por algo. No obstante, bien podría ser que se defienda el tercer significado del que habla Bobbio sin negar un criterio deductivo (como sería, por ejemplo, indicar que no hay lagunas ni contradicciones porque existe un sistema jerárquico establecido por una norma fundamental de estatus superior) o inductivo (como en el evento de sostener que no hay contradicciones ni lagunas porque en el ejercicio judicial de previsibilidad de lo que es derecho así se contempla).

8 Bobbio, op. cit., nota 4, p. 183.

9 Recordemos que el Kelsen de la primera edición de la Teoría pura del derecho negaba la existencia de lagunas auténticas y técnicas, considerando que son meras incongruencias entre lo que ordena y el derecho y lo que le gustaría fuera derecho al operador jurídico. Sin embargo, esta postura ha sido moderada por el propio Kelsen con posterioridad, así como por varios positivistas analíticos contemporáneos (véase la nota inmediatamente siguiente). 
Este tercer criterio, que nos recuerda de alguna manera el concepto tradicional de sistema en otras disciplinas, se centra en el concepto de orden como la prueba reina de la existencia de un sistema jurídico, de manera tal que si la teoría general del derecho dice - discursivamente- que en lo jurídico no hay lagunas (en virtud del principio de plenitud hermética del derecho) ${ }^{10}$ o es identificado - discursivamentecomo un sistema en orden jerárquico, entonces el derecho - en la realidad - es un sistema.

Pero justamente este aspectoe, según los autores del presente trabajo, parece un salto al abismo, en la medida que los sistemas de primer orden no corresponden a patrones de tal logicidad. Los trabajos tan adelantados de la sistémica en varios campos del saber apuntan a la imposibilidad real de tal tipo de sistemas.

Igualmente, los sistemas de segundo orden, es decir, elaboraciones o construcciones de explicación o modelización de otros sistemas, tampoco pueden ser entramados con respuesta lógica previsible en su totalidad. ${ }^{11}$

Cuando la teoría del derecho dice que en él no existen lagunas, o cuando la dogmática identifica al derecho en el mismo sentido, estamos frente a un sistema de segundo orden. Lo que es problemático es que un sistema de segundo orden es pretendido como el mismo sistema de primer orden, lo que significa que el discurso pretende reemplazar el derecho mismo. ¿Esto es posible? Creemos que no.

La única manera, siguiendo los planteamientos de lo que es un paradigma sentados por Jun, ${ }^{12}$ de que un sistema de segundo orden sea de logicidad y previsibilidad del rango deseado por los defensores del tercer tipo de concepción de sistema, es que haya un consenso tal entre las elaboracio-

10 Aspecto central en el pensamiento kelseniano, especialmente el que es fruto de la primera edición de la Teoría pura del derecho; Kelsen, Hans, La teoría pura del derecho, trad. Luis Legaz, México, Colofón, 2002, pp. 65-70 (parágrafos 41-43).

11 Véase François, International Encyclopedia..., cit., nota 3, t. 1, p. 347, núms. 1342 y 1343, citando a G. Bateson y M. Maruyama.

12 Kuhn, Thomas, La estructura de las revoluciones científicas, trad. Agustín Contin, México, Fondo de Cultura Económica, 1980, pp. 33-50. 
nes jurídicas y los dogmáticos en el mismo sentido. Bastaría mostrar entonces una contradicción en el subsistema normativo para probar que sí hay elementos no-ordenados en el sistema. ¿Es posible encontrarlos? Sí, y se expondrán dos casos hipotéticos pero no por ello alejados de la realidad judicial: 1) ¿qué sucedería si una norma de inferior categoría transgrede una norma superior y el órgano encargado de velar por la armonía material del sistema normativo sentencia que no hay tal violación? Pensemos el caso en que una ley ordena una sanción determinada y el juez que aplica dicha norma establece una sanción diferente y la sentencia es ratificada por las instancias respectivas haciendo tránsito a cosa juzgada; o cuando un decreto viola abiertamente un mandato legal pero el tribunal encargado de velar por la supremacía legal, por el motivo que fuere, sentencia que no hay tal violación dejando en firme la norma, lo que implica una sentencia contra legem. En este caso subsistirán dos normas contradictorias (la sentencia y la norma, la ley y la Constitución, en los ejemplos antes dados), sin que pueda decirse que una dejó de ser jurídica. ${ }^{13}$ 2) ¿En un sistema es posible encontrar elementos armónicos entre sí? No existe sistema sin posibilidad de movimiento, lo que, a su vez, supone contradicción y conflicto como motor de supervivencia, cosa que no sólo ha detectado Luhmann ${ }^{14}$ sino más recientemente los sistemistas jurídicos $\mathrm{Ni}$ -

13 Kelsen señala, para evadir este dilema, que las normas superiores tienen un carácter de alternatividad (especialmente las constitucionales), con lo cual las disposiciones inferiores toman decisiones que nunca supondrán una contradicción dentro del sistema. Pero esta salida no es más que un llamado ingenuo, puesto que el propio Kelsen señala que el juez de última instancia tiene el poder de crear una norma individual con el contenido previsto en la norma superior preexistente o con otro contenido distinto por él mismo determinado. Las críticas a la teoría de la alternatividad en Guibourg, Ricardo, Derecho, sistema y realidad, Buenos Aires, Astrea, 1986, pp. 52-54.

14 Las contradicciones desestabilizan el sistema y hacen patente esta desestabilización de la inseguridad de la expectativa, pero dicha desestabilización es funcional. Los sistemas complejos necesitan de un grado bastante alto de inestabilidad para poder reaccionar frente a sí mismos y a su entorno, y tienen que reproducir continuamente estas inestabilidades, pues de lo contrario desaparecerían. Luhmann, Sistemas sociales..., p. 332. 
kolaos Intzessiloglou ${ }^{15}$ y Ernesto Grün, ${ }^{16}$ entre otros. Pensemos el caso de un choque entre principios constitucionales que pone en evidencia la contradicción normativa: una persona accidentada y recluida en un hospital estatal, por motivos religiosos no da la autorización para una transfusión de sangre que es considerada como necesaria e inevitable. En este caso, ¿debe primar la libertad religiosa o el derecho a la vida? ¿Acaso no se evidencia una contradicción normativa-constitucional que no puede resolverse con los criterios tradicionales de solución de conflictos legales como lo son el jerárquico, el especial y el temporal? Un derecho fundamental no es superior a otro, pues ambos están consagrados en la propia Constitución, además de aceptarse tal cosa se estaría ante una tiranía que tanto atacó N. Hartmann; un derecho fundamental no es más especial que otro; un derecho fundamental no es anterior o posterior al otro. En fin, se requiere la ponderación que no elimina el conflicto en el seno del derecho sino que establece órdenes de precedencia en el caso concreto.

Entonces, creemos que existen las lagunas y las contradicciones dentro del sistema jurídico, máxime hoy día con el renacimiento de posturas cercanas al iusnaturalismo que fundan sus esperanzas del cumplimiento del modelo constitucional estatal en jueces cada vez más libres frente al mandato legal en la aplicación de una razón práctica jurídica. ${ }^{17}$

15 Cabe resaltar de su obra los siguientes textos: Essai d'Identification de la totalité sociale du phénomène juridique en tant que système, Berlín, Rechtstheorie Beiheft 10, 1986. "Stability and Change in Law; the Dynamic Equilibrium between the System of Law and its Social Environment”, 31 St Annual Meeting of the International Society for General Systems Research, Budapest, 1987. "L'approche systemique à Système ouvert comme stratégie d'Elaboration d'un projet d`Etude interdisciplinaire du phénomène juridique”, European Congress on System Science, Lausanne, 1989.

16 Grün hace una descripción de los trabajos académicos que intentan aplicar al mundo jurídico las elaboraciones sistémicas y cibernéticas. Grün, Ernesto, "La aplicación de la sistémica y la cibernética al derecho", en Botero Bernal, Andrés y Estrada Vélez, Sergio Iván (comps.), Temas de filosofía del derecho, cit., nota 2.

17 Una defensa de este modelo en Estrada Vélez, Sergio Iván, Los principios jurídicos y el bloque de constitucionalidad, 2a. ed., Medellín, Universidad de Medellín, 2006. Una crítica a este modelo en Botero Bernal, Andrés, "Ensayo sobre la crisis 
Este modelo, de gobierno de los jueces, ${ }^{18}$ amplía el rango de interpretación discursiva y aplicación fáctica del derecho que termina siendo imposible sostener en ella la existencia de un orden jerárquico estático que niegue las lagunas. ${ }^{19}$ Es explicable así porque ese positivismo fuerte de negación de la contradicción se enfrenta a la postura neoconstitucional que tanto la maximiza para poder predicar la libertad judicial frente a los mandatos tomados como superiores y jerárquicos por la exégesis. ${ }^{20}$

Si bien los ejemplos anteriores no son los únicos que permiten hablar de un sistema jurídico diferente al tercer modelo planteado por Bobbio, ya puede afirmarse que hay algo que no ha sido atrapado en aquella clasificación tan citada en la ciencia jurídica. ¿Estamos entonces frente a un cuar-

de la razón jurídica: Formalismo versus principios y/o valores", Opinión Jurídica: Revista de la Facultad de Derecho de la Universidad de Medellín, vol. 5, núm. 9, enero-junio de 2006, pp. 33-61.

18 Problema que no es nuevo: ya Lambert trabajaba críticamente sobre este concepto, en 1921, siguiendo de cerca el debate en torno al poder contramayoritario de los jueces estadounidenses que impidió en no pocas oportunidades se implantara, a principios del siglo XX, una legislación social (al respecto, véase la crítica de R. Pound a la Corte Suprema de su país por este aspecto, en: Pound, Roscoe, "Causes of Popular Dissatisfaction with the Administration of Justice", American Law Review, 40, septiembre-octubre de 1906, pp. 729-749. Lambert, Edouard, Il governo dei giudici e la lotta contro la legislazione sociale negli Stati Uniti, Milán, Giuffrè, 1996.

19 Claro está que no todas las teorías jurídicas contemporáneas que aceptan cierto protagonismo judicial defienden la existencia de lagunas en el derecho (por lo menos no en el derecho por principios). Entonces, no puede establecerse de manera absoluta una relación entre discrecionalidad judicial y lagunas normativas. Pero muchas de estas teorías (Dworkin, Alexy, Zagrebelsky, etcétera) consideran que el derecho por reglas sí presenta lagunas y contradicciones, lo que justifica la intervención del derecho por principios. Pero es justo aquí cuando estas posturas se separan en lo atinente a la discrecionalidad judicial, de manera tal que Dworkin negará la discrecionalidad en el derecho por principios en tanto cree que es posible una unidad de decisión correcta por parte del juez. En cambio Alexy aceptará las contradicciones entre principios de manera tal que se requiere la razón práctica como un regulador de las "lagunas de indeterminación" y conflictos propios del derecho por principios. Véase Zagrebelsky, Gustavo, El derecho dúctil: ley, derechos, justicia, Madrid, Trotta, 1999, capítulo "Derecho por principios". Alexy, Robert, El concepto y validez del derecho, Barcelona, Gedisa, 1994, pp. 159-174.

20 La discrecionalidad judicial y la aceptación de posibles conflictos entre las normas infraconstitucionales con la propia Constitución, son paradigmas comunes al neoconstitucionalismo. Al respecto, véanse los diversos trabajos compilados en Carbonell, Miguel (ed.), Neoconstitucionalismo(s), Madrid, Trotta, 2003. 
to modelo de sistema para el derecho? Creemos que sí. Este cuarto modelo de sistema no es otro que el planteado por la TSCD, con las características que a continuación enunciaremos.

\section{CARACTERÍSTICAS DE LA TSCD}

Son varias las características que la TSCD puede observar en el sistema jurídico, entendido éste como un cuarto modelo de lo que es sistema para el derecho.

A continuación expondremos algunas de esas características, pero de ninguna manera se trata de una enunciación taxativa.

\section{El sistema jurídico no se agota en el subsistema normativo (es decir, no se limita a un deber ser expresado según una estructura lógica determinada)}

Esto conlleva a considerar que el ordenamiento jurídico no es solo ordenamiento normativo. Entonces la mirada sobre el derecho no se agota en la "cascada normativa" (que, no obstante, no puede ignorarse por su importancia explicativa, al menos, para los sistemas jurídicos nacionales), ${ }^{21}$ pues abarca otros tópicos como los siguientes: 1) las relaciones entre los subsistemas jurídicos entre sí (intorno);22

21 Tal como lo ha señalado el profesor Enrique Cáceres, se están revisando los cimientos mismos del propio paradigma analítico, básicamente el concepto de regla de reconocimiento de Hart, puesto que dicho concepto es circular e indeterminado, y que fue generado para dar cuenta de los derechos estatales, pero adolece de importantes insuficiencias frente al fenómeno de la globalización. En este punto es donde aparece un primer encuentro entre el enfoque sistémico y la jurisprudencia analítica, pues en la propuesta teórica que se está trabajando el concepto de redes institucionales, concebidas desde la perspectiva de las redes sociales, constituye la piedra de toque para determinar un nuevo criterio de membresía apto para los tiempos de globalización. En fin, el positivismo (incluso en analítico, debe reformularse ante los nuevos tiempos políticos, económicos y culturales. Agradecemos al profesor Cáceres por su ayuda en la redacción de esta nota.

22 El intorno, noción acuñada por François que caracteriza como el conjunto de los subsistemas y sus interrelaciones, el cual constituye el entorno común de los subsistemas dentro del sistema. Véase François, Diccionario..., cit., nota 3, pp. 97 y 98. 
2) las relaciones entre el sistema jurídico con otros sistemas (entorno); 3) la función de los diferentes subsistemas jurídicos; 4) la estructura del sistema jurídico; como los más importantes.

Esto nos lleva a considerar al positivismo normativista o estructuralista, al realismo funcionalista y al iusnaturalismo como miradas insuficientes del derecho, observaciones parcializadas, fragmentadas, ignorando así un complejo andamiaje, como el que presenta el derecho en el mundo globalizado de este siglo XXI. Pero lo más grave de esas posturas teóricas es que tienden a considerar su punto de visión como el único existente, de manera tal que para el formalismo jurídico colombiano ${ }^{23}$ (escuela del positivismo normativista) el sistema jurídico se reduce al subsistema normativo, o cómo para el iusnaturalismo axiológico el subsistema normativo está en una relación jerárquica preestablecida con unos valores identificados como superiores por esta misma escuela conduciendo a otro reduccionismo igual de peligroso que el del formalismo.

23 El formalismo jurídico ha sido definido como la pretensión francamente positivista de distinguir el derecho del no derecho sin hacer referencia al contenido de las reglas jurídicas (Bobbio, Norberto, El problema del positivismo jurídico, 6a. ed., México, Fontamara, 1999. p. 84). No obstante, el formalismo jurídico latinoamericano si bien parte de esa definición, se caracteriza además por su apego a las fórmulas, la forma por la forma, el tecnicismo, la eficacia simbólica, etcétera. Un estudio (histórico y analítico) de dicha teoría sui generis en López, Diego, Teoría impura del derecho: la transformación de la culturajurídica latinoamericana, Bogotá, Universidad de los Andes, Legis y Universidad Nacional de Colombia, 2004. "El formalismo jurídico en Colombia se ha ido construyendo lentamente a lo largo de los siglos XIX y XX. Hoy el formalismo local está formado, en una mezcla compleja y quizá inestable, por la recepción de tres transplantes iusteóricos distinguibles: primero, la recepción en Colombia del Code Napoleón y una tecnología formalista de lectura del mismo predominante en el siglo XIX francés... segundo, el impacto que tuvo el trasplante de los métodos de los romanistas y privatistas alemanes del XIX... y tercero, ... la teoría pura del derecho". Ibidem, p. 130. Llama la atención que López ignora en esta cita el componente heredado de la colonia y de la independencia en la configuración del formulismo local, puesto que el Código Civil entra a Colombia en la segunda mitad del XIX (Botero Bernal, Andrés, Estudio de la constitución antioqueña de 1812: modelo de lectura en tres actos del constitucionalismo provincial hispanoamericano, Medellín, Universidad de Medellín, 2007, en prensa). Debe destacarse que el formalismo colombiano es una categoría que no se presenta necesariamente en otros países hispanoamericanos. 
En conclusión, el derecho no se agota como sistema en lo normativo, pero no prescinde de él, tal como ya lo había señalado el tridimensionalismo. En consecuencia, las miradas estructuralistas están limitadas en su observación sistémica. Pero de igual manera, las miradas no estructuralistas están también parcializadas en tanto tienden a minimizar las relaciones de complejidad entre los susbsistemas para terminar privilegiando uno sobre los otros.

Además, el sistema jurídico, al ser un sistema complejo compuesto de elementos de distinto origen y función (como normas, hechos, valores, etcétera), implica que sus agentes se vean abocados a tomar decisiones o a resolver situaciones teniendo en cuenta dicha complejidad. Así, el legislador, cuando se aboca a proyectar una norma, procede sistémicamente, al igual que el abogado que presenta la defensa de su cliente. ${ }^{24}$ Es decir, en todos estos casos el sistema que están usando está integrado por estos elementos y lo que importa a una TSCD son las interrelaciones que se establecen entre ellos y con el entorno, pues como lo expresó Botero:

...el derecho entendido como una especialización sin fragmentación no es más que una confluencia de una postura sistémica compleja (que reconoce la interacción de lo que estudia con lo biológico, lo social y lo ambiental), interdisciplinaria (e incluso transdisciplinaria) y constructivista (metodología de investigación basada en problemas). ${ }^{25}$

2. El sistema jurídico implica las intenciones y los deseos de conducción de la sociedad

Entonces el sistema jurídico no es sólo cómo "en verdad" opera el derecho, sino que hay que reconocer que hay elementos axiológicos que intentan conducir a la sociedad a través del derecho mismo a otros escenarios (es decir, no se ago-

24 Véase Cueto Rúa, Julio, Judicial Methods of Interpretation of the Law, The Publications Institute Paul M Hebert, Law Center Louisiana State University, 1981, capítulo II.

25 Botero, "Nuevos paradigmas científicos y su incidencia en la investigación jurídica”, Diálogos de Saberes, Bogotá, núm. 18-19, enero-diciembre de 2003, p. 171. 
ta en el ser del deber ser). De esta manera, el derecho no sólo describe conductas sino que además las valora (teoría de la ciencia bifronte), ${ }^{26}$ lo que se logra, obviamente, con la relación entre el sistema jurídico y los sistemas político y moral.

Pero ese aparato descriptivo (descriptivo para el investigador, en tanto lo ve como un objeto con información contenida en sí misma, como lo sería, por ejemplo, el decir que $A$ es delito y que $B$ es lícito), implica una conducta que se desea no se realice o que se realice (ya sea eficacia material o eficacia simbólica). ${ }^{27}$ La parte valorativa del derecho no es neutral, ni moral, ni política; entonces, el derecho co- mo sistema actuante en la sociedad, no es puro frente a los otros sistemas con los que se comunica.

\section{El sistema jurídico no se agota en valoraciones de cómo queremos que sea la sociedad o cómo queremos que sea el derecho (es decir, no se agota en el deber ser del deber ser)}

El derecho cumple de manera compleja funciones de ser y deber ser en varios grados. ${ }^{28}$ Sirva exponer un caso: Una norma $\mathrm{X}$ tiene una información como lo sería, por ejemplo,

26 Concepto desarrollado en Guibourg, Ricardo, Derecho, sistema y realidad, Buenos Aires, Astrea, 1986. Según él, el derecho tiene las funciones de motivar e interpretar conductas, pero cada una de estas funciones apunta a una ciencia del derecho distinta e incompatible con la requerida por la otra. Partiendo de este concepto consideramos pues que el derecho es una ciencia bifronte, en tanto busca modificar un entorno siendo además su producto. El derecho es fruto de una sociedad y cumple una labor legitimadora de lo existente, en un proceso de descripción de conductas, pero no se niega a sí mismo la búsqueda de modificar la misma estructura que lo produce. Puede operar tanto como control de los ciudadanos frente al poder, como sistema de control del poder en defensa de los ciudadanos. En fin, con la ciencia bifronte se plantea una interacción entre el estructuralismo y el funcionalismo para una mejor comprensión del derecho.

27 Véase Botero, Diagnóstico de..., cit., nota 2, pp. 33-45.

28 No nos referiremos a la falacia humeana (que del ser no puede derivarse un deber ser). Creemos que el argumento de Hume con el que ataca al derecho natural se hace con otras significaciones diferentes a las que usaremos en este apartado. Además, "ser" que será entendido como descripción y "deber ser" que será significado como valoración, no son categorías absolutas ni puras. No hay una descripción pura, pero sí hay juicios descriptivos. Igual sucede con los juicios valorativos. 
decir que quien matare a otro incurrirá en una sanción penal. Para el investigador, esta información es una descripción, pero igualmente hay un deber ser en su esencia (quien matare a otro deberá incurrir en pena de prisión), lo que es más claro para el jurista. Pero además, la mirada que sobre el derecho mismo se hace puede ser sobre cómo es el derecho en un momento y en un lugar concreto, o cómo debe ser el derecho en un momento y en un lugar concreto. Entonces hay una visión descriptiva como una valorativa del mismo. Esto nos lleva a ver que hay un ser/deber ser que a su vez puede ser visto desde el ser o desde el deber ser. Pero incluso, podría pensarse en cómo el debe ser el deber ser del deber ser (sería el caso de juzgar a la doctrina por sus consideraciones de cómo debe ser el derecho mismo desde su faceta valorativa - caso en que se critica una escuela jurídica justo en lo que propone debería ser el derecho-).

Otro ejemplo podría aclarar este aspecto, ahora no con una norma sino con todo un sistema jurídico. El sistema jurídico argentino es una realidad social que puede ser descrita (contemplación desde el ser) a la vez que es en sí mismo un conjunto de postulados deontológicos (deber ser). Pero además podemos valorar ese sistema jurídico nacional para señalar si nos gusta o no, que dicho con otras palabras no es más que afirmar cómo debe ser (para nosotros) ese deber ser (faceta deontológica o normativa del derecho fruto del hecho de que son mandatos) - ser (en tanto el derecho nacional es una realidad descriptible, que constituye la faceta funcionalista-realista). De esta manera se está dando lugar a valoraciones (faceta moral del derecho desde la óptica de un observador que generalmente es un operador jurídico). Pero esa misma valoración moral del derecho argentino (que es un deber ser del deber ser - ser) puede ser descrita por un investigador (ser del deber ser del deber ser-ser) o nuevamente valorada por un operador jurídico (deber ser del deber ser del deber ser - ser del derecho nacional argentino). 


\begin{tabular}{|c|c|c|c|c|}
\hline \multirow{5}{*}{ Derecho } & \multirow{3}{*}{$\begin{array}{l}\text { 1. Deber ser (va- } \\
\text { loración sobre el } \\
\text { derecho). }\end{array}$} & \multirow{2}{*}{$\begin{array}{l}\text { 1.1. Deber ser del deber ser del } \\
\text { derecho (ejemplo: cómo cree el } \\
\text { operador jurídico debe ser el ideal } \\
\text { de una institución jurídica). }\end{array}$} & \multirow{2}{*}{$\begin{array}{l}\text { 1.1.1. Deber ser del de- } \\
\text { ber ser del deber ser del } \\
\text { derecho (ejemplo: valorar } \\
\text { las propuestas morales } \\
\text { de un autor sobre el } \\
\text { ideal de una norma jurí- } \\
\text { dica) }\end{array}$} & $\begin{array}{l}\text { 1.1.1.1. Deber ser del } \\
\text { deber ser del deber ser } \\
\text { del deber ser del dere- } \\
\text { cho. } \\
\begin{array}{l}\text { 1.1.1.2. Ser del deber } \\
\text { ser del deber ser del } \\
\text { deber ser del derecho. }\end{array}\end{array}$ \\
\hline & & & & \\
\hline & & $\begin{array}{l}\text { 1.2. Ser del derecho ser del de- } \\
\text { recho (ejemplo: un operador ju- } \\
\text { rídico que describe la idea de } \\
\text { una institución jurídica). }\end{array}$ & & \\
\hline & 2. Ser (descrip- & $\begin{array}{l}\text { 2.1. Ser del ser del derecho } \\
\text { (ejemplo: una descripción de al- } \\
\text { guna teoría que ha descrito a su } \\
\text { vez el derecho nacional). }\end{array}$ & & \\
\hline & ción del derecho). & $\begin{array}{l}\text { 2.2. Deber ser del ser del dere- } \\
\text { cho (ejemplo: Valorar una des- } \\
\text { cripción de lo que es derecho en } \\
\text { un momento determinado). }\end{array}$ & & \\
\hline
\end{tabular}


$\mathrm{El}$ anterior cuadro aclara este análisis que es una herramienta útil al momento de distinguir los diversos grados y las diversas cualidades de los juicios que en torno al derecho (o en torno a una norma o institución jurídica) pudiesen hacerse.

Este esquema, que puede prolongarse hasta el infinito, logra una gran precisión en nuestras observaciones (pues bien podría hacerse una descripción de una teoría que propugna por un cambio de una norma, es decir, ser del deber ser del deber ser), y permite sostener que las valoraciones de cómo queremos que sea el derecho no lo agotan, pero tampoco le son ajenas.

En consecuencia, cuando se describe y/o se valora el derecho (o una norma o una institución jurídica), esto puede hacerse en grados hasta el infinito, pero siempre, en cualquier escalón, hay un observador que describe y/o valora que es determinante en su observación que es igualmente gradual.

Esto nos lleva a considerar en este aspecto, como en otros expuestos en el presente trabajo, que, como ha señalado Heinz von Foerster, el observador influye sobre lo observado, ${ }^{29}$ que el sistema incluye a quien lo observa.

4. El sistemajurídico no es puro, ni mucho menos puede estudiarse como si lo fuera, en tanto es un sistema abierto al entorno y en continuo movimiento

Esto supone hacer un énfasis en el tema del movimiento, de las temporalidades, de su evolución, lo que ha sido desconocido por varias de las tesis jurídicas generales que han visto o creído ver un sistema jurídico estático, inmóvil, como una fotografía; o, a lo sumo, como un sistema dinámico pero cuya movilidad se da hacia dentro, es decir, creer que la ciencia del derecho no requiere algún referente exter-

29 Véase Foerster, Heinz von, Observing Systems: Selected Papers of Heinz von Foerster, Seaside, CA, Intersystems Publications, 1981. 
no para explicar la movilidad intrajurídica. ${ }^{30}$ La sistémica o teoría general de sistemas hace énfasis en la dinámica de todos los sistemas, sobre la base de la cibernética (retroalimentación, bifurcaciones, caos, etcétera), ${ }^{31}$ siempre vinculado al entorno.

También esta característica supone hacer algunos comentarios en torno a su función autopoiética entendida como autoorganización. ${ }^{32} \mathrm{El}$ derecho es autopoiético o autoorganizado siempre y cuando se entienda que tal función no implica un cierre del sistema frente al entorno. Claro está que como modelo de segundo orden, y desde un ámbito formal, podemos explicar sin necesidad de referentes externos la movilidad normativa, 33 pero esta postura presenta varios límites: 1) El propio Kelsen reconoció que, en ciertos momentos excepcionales, la nomodinámica obedece a situaciones del entorno, como por ejemplo la revolución, la costumbre y el desuso, en las cuales la entrada o salida de una norma del sistema jurídico depende de la eficacia (esto

30 Kelsen se refirió a la pureza del derecho para efectos esencialmente epistémicos, esto es, un sistema cerrado para lo que concierne a la estructuración de una disciplina científica. Mal sería considerar que Kelsen creía en un sistema jurídico cerrado ontológicamente hablando. Esto es, no negaría la vinculación sociedad derecho, que sería pertinente para el sociólogo, aunque intrascendente para el científico del derecho. Esta propuesta epistémica da un importante valor a la nomodinámica, esto es, a la movilidad normativa a su interior, sin embargo considera que no se requiere algún elemento extrasistémico para dar cuenta de tal dinamismo. Esto vendría siendo, en términos de Luhmann, una autopoiesis propia de un sistema - epistémicamente- cerrado (ver la nota 34).

31 Véase Grün, Ernesto, Una visión sistémica..., Lexis Nexis, cit., nota 1, pp. 21-23, capítulo X y bibliografía allí citada.

32 El profesor Grün preferirá hablar de autoorganización en vez de autopoiesis. El profesor Botero prefiere en cambio el concepto de autopoiesis al de autoorganización. No obstante, ambos toman distancia del concepto de autopoiesis (y de sus consecuencias) en el ámbito jurídico que pregona Luhmann. Cfr. Luhmann, Niklas, Autopoietc Law: A New Approach to Law and Society, Berlin, Walter de Gruyter, 1988. pp. 12-36 y 335-348.

33 Véase respecto de la nomodinámica kelseniana y la autopoiesis: Schmill, U., "El modelo del mandato, el orden jurídico y las normas de competencia" (en línea), Cuadernos Electrónicos de Filosofía del Derecho, núm. 5, 2002; http://www. uu.es/CEFD/5/schmill.html. Kelsen (especialmente el de la primera edición de la Teoría pura del derecho) podría ser leído desde la sistémica autopoiética de Luhmann. 
es, de una acción y omisión social). 2) Cuando el observador no sólo se pregunta por la forma de operar la movilidad en situaciones no excepcionales, esto es, en el desenvolvimiento cotidiano del derecho estatal (que por cierto ya es una presupuesto ideal), sino que además se interroga por el contenido de esas normas, tiene que incluir en sus descripciones-valoraciones el entorno. 3) El procedimiento nomodinámico tiene en su fondo una apuesta no (exclusivamente) jurídica, como lo es el Estado, el legislador, el constituyente, etcétera.

Así, si se cree que ser autopoiético o autoorganizado supone un proceso de autocreación sin necesidad del entorno, el derecho no tendría tal cualidad. El derecho es un subsistema social (que bien podría ser asemejado funcionalmente al inmunológico en el sistema biológico) por lo cual su proceso de autoconstrucción no es ajeno al entorno social (relación con los otros subsistemas) ni al entorno que rodea lo social en su sentido más amplio.

Este proceso autopoiético o autoorganizado abierto, si se nos permite usar tal expresión, pone de relieve que el derecho no es, sistémicamente hablando, un sistema puro pues no puede prescindir en su conformación de lo político, lo social, lo moral, etcétera. Además, un sistema de segundo orden, una modelación, tampoco es neutro. Entonces, ni en un primer grado, ni en uno segundo, el derecho es puro en tanto como fenómeno social no es un entramado absolutamente diferenciable, y como modelo no se podría pensar en planteamientos jurídicos sin ideologías $o$ sin cogniciones intencionadas.

Esto es consecuente con afirmar que en el enfoque sistémico la identidad del sistema se la da el observador o el operador jurídico. Como lo hemos señalado al comienzo de este trabajo "la condición de sistema no es una cualidad intrínseca de las cosas, sino una actitud o apreciación de cada uno, por lo que cuando decimos que tal cosa concreta o abstracta es un sistema deberíamos decir que la consideramos un "sistema". 
5. Si observamos el complejo panorama de la globalización con sus diversos procesos que, desde hace algunos decenios, se están produciendo en la esfera de lo jurídico y que se están desenvolviendo con pautas muy disimiles y aun contradictorias, a velocidades muy diferentes y con propósitos propios en los diversos ámbitos, desde el ángulo de la filosofía y la teoría del derecho se hace sumamente dificil, casi utópico, pensar en una teoría general, clara y sistemática (en términos de jerarquía preconcebida o estática) como la que se pretendía construir en el siglo pasado

Hoy día estamos asistiendo a lo que podríamos caracterizar como una auténtica mutación genética del derecho: cambian los actores del proceso jurídico, cambian las modalidades de producción y funcionamiento de las reglas jurídicas; en fin, lo jurídico se transforma radicalmente. De esta forma, debemos tener presente que cambia la epistemología del derecho en general, pero en particular cambia de episteme el derecho forjado en la tradición iuspositivista, tradición que ha sido, de alguna manera, la que originó la creencia en la posibilidad de crear una concepción unitaria, general, clara y jerárquica del derecho, creencia que se intentó materializar durante años en los estudios de "teoría general del derecho". Esto nos permite afirmar que se podría hablar de sistema jurídico en singular si lo que se pretende es hacer una TSCD como modelo (sistema de segundo orden) que identifique patrones estructurales y funcionales relativamente comunes, pero la TSCD más que identificar esos patrones comunes buscaría plantear un nuevo lenguaje que habilite al investigador para una lectura más eficiente de los sistemas jurídicos, éstos sí en plural. En otras palabras, creer en una teoría general del derecho que permita la precomprensión de los sistemas jurídicos particulares es un gran error epistemológico, por falla tanto en el discurso epistemológico como por incapacidad de 
comprender la movilidad de la propia episteme, de los sistemas jurídicos dinámicos por naturaleza.

Por otra parte, la idea de una "teoría general del derecho" surge en una época eurocéntrica, en el siglo XX. Pero hoy en día, en un mundo mucho más complejo e interrelacionado, en que adquieren relevancia sistemas jurídicos de tan disímil estructura como la de muchos países mahometanos (con su aplicación de la sharía, por citar un caso), el sistema jurídico de China, los novedosos sistemas jurídicos propios del mundo globalizado, como la lex mercatoria, la lex retis, entre otros, se hace problemático seguir hablando de una "teoría general" que crea a pie juntillas que no hay mayor variabilidad o deriva genética en los sistemas jurídicos.

Pero si bien es cierto la existencia de múltiples sistemas, de movilidad, de temporalidad y de evolución, esto no implica, como ya se dijo, que no se pueda hablar de una TSCD siempre y cuando ésta se caracterice justamente en (i) negar la posibilidad de creer que los sistemas pueden ser comprendidos a priori, y (ii) en rechazar la opción de una teoría que justifique, explique o comprenda (según el paradigma que se adopte) todos los sistemas presentes. La TSCD partiría de la negación de un solo sistema, y por tanto de la imposibilidad de un solo sistema de comprensión o sistema de segundo grado; pero ya afirmar que no hay un solo sistema de comprensión ya es UNA teoría que puede ser aplicada, por lo menos en este aspecto, a todos los sistemas. Entonces, en conclusión, bien puede hablarse de teorías sistémico-cibernética-jurídicas o una teoría general sistémico-cibernética-jurídica, dependiendo todo del punto de vista asumido y del campo de investigación que nos planteemos.

Esto nos remite a lo que escribió el profesor Enrique Cáceres Nieto:

...no es verdad que una supuesta estructura de la realidad determine nuestras teorías, sino más bien nuestras teorías las que determinan lo que podemos construir como realidad. La teoría general de sistemas constituye una manera especí- 
fica de estructurar el mundo, con su propia ontología de constructos conceptuales, la relación establecida entre ellos y su propia metodología, a partir de la cual se obtienen modelos desde los que se concibe la realidad de cierta forma. Sin embargo, a diferencia de aquéllas, la teoría general de sistemas no constituye una materia acotada que se ocupe de un grupo particular de fenómenos, como ocurre con la química o la física; ni tampoco es una materia que haya surgido de la superposición de problemas preexistentes como sucede con la bioquímica. La teoría general de sistemas constituye una metadisciplina cuya materia virtual es prácticamente cualquier otra disciplina. Su mérito principal consiste en permitir elaborar infinidad de modelos explicativos en prácticamente todas las áreas del conocimiento con una gran economía conceptual y reglas de procesamiento de la información de tipo metodológico que le son propias. Como sucede con toda teoría, las explicaciones resultantes de aplicar la teoría general de sistemas son el producto de constructos conceptuales de los que se predican ciertas propiedades, funciones o leyes, que se atribuyen a las entidades con las que se instancia la teoría al momento de elaborar explicaciones o modelos. ${ }^{34}$

Y como nos lo ha señalado él mismo, es en este aspecto donde se encuentra la piedra de toque de la necesidad de hacer un viraje sistémico, es decir, usando terminología clásica podría decirse que el explanadum ha mutado y por lo tanto se requiere de un explanans diferente. ${ }^{35}$

\section{El sistema jurídico es dinámico, pero dentro de su dinamismo se generan jerarquías no estáticas}

Esto es consecuencia de aceptar que en el sistema jurídico hay lagunas, hay vacíos, hay conflictos, hay entropia. Pero la aceptación de cierto desorden, en los términos ante-

34 Cáceres Nieto, Enrique, “Prólogo”, en Grün, Ernesto, Una visión..., cit., nota 1, Buenos Aires-México, p. XVI-XVII.

35 Cáceres Nieto, Enrique, comunicación personal. 
riores, no supone hablar de la inexistencia de jerarquías en varios de los subsistemas jurídicos. Así, por ejemplo, en el subsistema normativo, existe una fuerte creencia de los operadores jurídicos, sustentada en varias premoniciones normativas, en que no todas las normas tienen el mismo rango de aplicabilidad. Entonces, el movimiento del subsistema normativo mismo (movimiento que supone una interrelación con otros subsistemas jurídicos y con el entorno del sistema jurídico) supone la aparición de jerarquías (asunto que de alguna manera trabajó el realismo judicial escandinavo cuando analizó las jerarquías normativas a partir del proceso de aplicación judicial del derecho). ${ }^{36}$

Pero estas jerarquías que surgen en el sistema jurídico (entre los subsistemas) y especialmente en su subsistema normativo (entre sus elementos; por ejemplo: para muchos sistemas jurídicos contemporáneos la Constitución es superior a la ley), no son absolutas ni estáticas, pues desde el mismo momento en que una jerarquía se establece como definitiva en un sistema, éste se niega a su evolución. Las jerarquias cambian siguiendo patrones de temporalidades, y fruto no sólo de lo que DEBE SER el derecho (que supone, entre otras cosas, las partes valorativa y normativa) sino también que cambian conforme a lo que ES el derecho (que implica, especialmente, la parte descriptiva).

Esta visión dinámica de las jerarquías es aceptada incluso por tesis positivistas analíticas. Dicen así Bulygin y Mendonca: "Debemos tener presente, pues, que los sistemas jurídicos no son meros conjuntos de normas, sino estructuras jerárquicas; que existen ciertas relaciones jerárquicas entre las normas pertenecientes a un sistema jurídico"; luego afirman: "si por "sistema normativo" entendemos un conjunto ordenado de normas, entonces toda modificación de las relaciones ordenadoras modifica automáticamente el sistema

36 Ross, op. cit., nota 7, pp. 77-79. Esta visión jerárquica es duramente criticada por Guibourg en Guibourg, Derecho..., cit., nota 13, pp. 66-68. 
mismo"; 37 pero, a diferencia de estos autores, creemos que la jerarquía no es la que aporta la dinamicidad al sistema, sino todo lo contrario.

En consecuencia, los conceptos del derecho se hallan estrechamente relacionados entre sí por razones de interdefinibilidad, como en la nomoestática kelseniana; pero también lo están en forma dinámica en la medida que el derecho contiene mecanismos que lo autorregulan y automodifican. Por tanto, la autorregulación depende de cierta forma de retroacción (feed-back), pues desde los propios mecanismos del derecho se advierte que algunos resultados no son normativamente adecuados (casos de contradicción, laguna o inconstitucionalidad) y esa información se transforma en acción correctora (derogación, modificación, interpretación, construcción jurisprudencial). El tejido del derecho se va regenerando constantemente, con lo que satisface la función de autopoiesis o de autoorganización.

Para quienes veían a los sistemas jurídicos como sistemas cerrados el problema de las llamadas "lagunas del derecho" resultaba poco menos que insoluble: o bien se negaba dogmáticamente su existencia, o bien se imaginaban mecanismos de interpretación para su llenado. Si el tema es pensado como abierto a un entorno, las lagunas serán cubiertas por elementos provenientes de ese entorno, así como el organismo vivo obtiene su alimento del ambiente.

7. El trialismo (en sus diferentes manifestaciones) dio elementos importantes para entender el derecho, pero no lo comprende como un sistema (por lo menos no como aqui concebimos un sistema)

El trialismo o tridimensionalismo, en cualquiera de sus manifestaciones hasta ahora presentes en el campo de la filosofia del derecho, ha dado grandes aportes, especialmente para la superación de encarnizados debates entre estructu-

37 Bulygin, Eugenio y Mendonca, Daniel, Normas y sistemas normativos, Madrid, Marcial Pons, 2005, pp. 79 y 80, respectivamente. 
ralistas, funcionalistas y iusnaturalistas. No obstante, el trialismo, si bien es un paso hacia la TSCD no logra llegar hasta lo que esta última propone: un cuarto modelo de sistema para la lectura de los sistemas jurídicos.

Resulta que el trialismo supone que el derecho es una sumatoria de preconcepciones de la realidad del derecho, de los valores jurídicos y de las normas. Pero dicha postura es fundamentalmente mecanicista, lo que hace creer que la comprensión de lo jurídico es un proceso causal de sumatoria, coherente, claro, disponible y planeable. Dicho en palabras del profesor Atienza:

Con ello (el trialismo) se trata de ofrecer un panorama del derecho más completo que el que resulta de cada una de las anteriores perspectivas, pero se corre el riesgo de incurrir en una especie de sincretismo jurídico en que la pérdida en cuanto a la nitidez que ofrecian las versiones parciales no resulte compensada por una visión que verdaderamente vaya más allá, esto es, que sea algo más que una mera yuxtaposición de elementos. ${ }^{38}$

En cambio, la TSCD considera que el derecho (como sistema de primer orden) no puede reducirse a tres esferas. La enunciación exacta del número de esferas depende de cada sistema jurídico en particular, por lo que en la TSCD sólo podrá afirmarse que no es posible una reducción a tres.

Entonces, el trialismo es una manera pedagógica de aprehender la complejidad del derecho. Pero el sistema jurídico es tan complejo que si se grafica no podría ser nunca una pirámide o triángulo (metáfora arquitectónica ${ }^{39}$ muy de moda entre los trialistas, especialmente los seguidores de Reale),

38 Atienza, Manuel, Derecho y argumentación, Bogotá, Universidad Externado de Colombia, 1997, pp. 22 y 23. Claro está que el profesor Atienza propondrá un enfoque que supere el trialismo: "Considerar al derecho como un intento, una técnica, para la solución de determinados problemas prácticos... en definitiva, a considerar el derecho como argumentación" (ibidem, p. 23).

39 Sobre las metáforas arquitectónicas en el derecho: Narváez Hernández, José Ramón, "Arquitectura jurídica: certeza y seguridad en el derecho", Revista Telemática de Filosofía del Derecho, núm. 6, 2002-2003, www.filosofiayderecho.com. 
metáfora, además, estática, donde se comunican tres facetas que agotan lo jurídico. En conclusión, el trialismo conduce a confusiones pues hace creer que el derecho es una sumatoria de tres elementos, ignorando la complejidad de las relaciones intrajurídicas y con el entorno. ${ }^{40}$

Dicho de otra manera, el derecho no se reduce en los análisis que desde la ciencia del derecho pueden hacerse ni desde la realidad de lo jurídico, a tres ámbitos: el normativo, el funcional y el valorativo. Si bien éstos ponen en evidencia la mayoría de las posturas iusfilosóficas hasta ahora planteadas (el estructuralismo, el funcionalismo y el iusnaturalismo), bien caben conexiones no fácilmente atribuibles en dichos ámbitos o en las teorías antes aludidas, como por ejemplo la pregunta por la vinculación entre los componentes de la biología humana con el derecho que no corresponde siquiera a los modelos funcionalistas preocupados más por una teoría de la eficacia jurídica.

Así, la TSCD evitará estos reduccionismos del derecho a un número determinado de ámbitos o de posturas teóricas, pues se parte que cualquier enumeración que se haga no podrá ser exhaustiva.

\section{La teoría sistémica disuelve buena parte de los debates entre iuspositivismo - iusnaturalismo- realismo}

Esto sería el gran aporte de la teoría sistémica a la filosofía del derecho, y sería una consecuencia teórica de la característica inmediatamente anterior (apartado 7). Si el derecho es un entramado complejo, mal se haría en un sistema de segundo orden (es decir, de un sistema de teorías y comprensiones sobre el derecho, como lo sería el iuspositivismo, el iusnaturalismo, el realismo, el trialismo, etcétera) reducir

40 Además, el trialismo ha tenido fuertes problemas metodológicos y epistemológicos, basados en la dificultad de ensamblar estas tres dimensiones entre sí sobre un soporte de pensamiento tradicional y analítico. Cfr. Grün, Ernesto, Una visión..., cit., nota 1, 2006, p. 30. 
el sistema analizado (es decir, el derecho mismo, que sería el sistema de primer orden) a ciertos valores, a la realidad, a la norma, etcétera. Esto lo explicamos con un ejemplo: si el iusnaturalismo teológico en su vertiente tomista considera una jerarquía entre ley eterna, ley natural y ley humana, esto es predicable de la construcción que ese sistema de segundo orden ha hecho; pero reducir el derecho mismo (es decir, el sistema de primer orden sobre el que se funda las teorías tomistas que sería un sistema de segundo orden) a la jerarquía ya anotada, sería un salto peligroso que niega la complejidad de ambos sistemas y la perspectiva límite que tiene todo sistema de segundo orden, perspectiva que puede enunciarse de la siguiente manera: una comprensión, una explicación, una teoría de un sistema, no puede abarcarlo íntegramente.

Entonces, sería validable en la ciencia jurídica un estudio que se centre en un solo interés, siempre y cuando ello no llegue al punto de considerar inexistentes otros intereses de segundo nivel también visibles en el sistema de primer orden.

De tal manera, si se acepta que el derecho es un sistema con interacción constante entre varios subsistemas y entre éstos con el entorno de lo jurídico, mal se haría en creer que el sistema se termina en una estructura normativa, en una función o realidad (ya sea judicial o social) o en unos principios o valores superiores emanados de la razón, de Dios, etcétera.

La TSCD sería en sí misma una forma, no de responder al interrogante normativista, funcionalista o iusnaturalismo, sino más bien de disolución de los problemas que dieron origen a estas tres escuelas que en no pocas oportunidades se autoexcluían.

Además, la TSCD pone en evidencia las relaciones determinantes entre el derecho con sus entornos más significativos (política, economía, sociedad, etcétera) desde un ángulo diferente y más preciso al aportado por la tradición iusfilosófica. Por otra parte permite observar los procesos de dese- 
quilibro, equilibrio dinámico, etcétera, que caracterizan los procesos jurídicos en el mundo actual, a la vez que incorpora el estudio y la aplicación de los nuevos sistemas jurídicos del mundo globalizado dentro de la filosofía y la teoría jurídicas.

En conclusión, la TSCD impediría radicalismos en los que se enfocarían en cómo debe ser el derecho, en los que se centran en cómo se desenvuelve el derecho y en los que se detienen en cómo se estructura el derecho (que incluye la pregunta de qué dice el derecho).

Como podemos vislumbrar a través de lo dicho hasta aquí, la TSCD resolvería o disolvería muchos de los problemas y debates actuales de la filosofía del derecho, a partir del cambio de paradigma. Pero aquí sólo enunciamos algunos de los problemas que resuelve o disuelve, pero estamos seguros que las ventajas en la comprensión que permite la TSCD son mucho más de las enunciadas en este trabajo.

\section{CONCLUSIONES}

La TSCD, a cuya concreción tiende el presente trabajo, constituirá, así lo esperamos, el paradigma que la complejidad de los temas y de los sistemas jurídicos (nacionales, internacionales, transnacionales, etcétera) requiere hoy en día para hacer frente a la crisis de su funcionamiento práctico y de su investigación científica y filosófica.

Las herramientas de la sistémica y la cibernética están a disposición, y creemos que serán de suma utilidad para efectuar esta tarea en un momento de grandes convulsiones mundiales en los ámbitos económico, social y político que justifican encarar de esta manera la lucha por el derecho que ya exigía Rudolf von Ihering hace más de un siglo.

\section{BIBLIOGRAFÍA}

ALEXY, Robert, El concepto y validez del derecho, Barcelona, Editorial Gedisa, 1994. 
ATIEnZA, Manuel, Derecho y argumentación, Bogotá, Universidad Externado de Colombia, 1997.

BoBBIo, Norberto, El problema del positivismo jurídico, 6a. ed., México, Fontamara, 1999.

— Teoría general del derecho, Bogotá, Temis, 1987.

Botero Bernal, Andrés, Diagnóstico de la eficacia del derecho en Colombia y otros ensayos, Medellín, Señal Editora y Fondo Editorial Biogénesis, 2003.

_ - "Ensayo sobre la crisis de la razón jurídica: (formalismo versus principios y/o valores)”, Opinión Jurídica, núm. 9, 2006.

—_, Estudio de la Constitución antioqueña de 1812: modelo de lectura en tres actos del constitucionalismo provincial hispanoamericano.

, "Nuevos paradigmas científicos y su incidencia en la investigación jurídica”, Diálogos de Saberes, Bogotá, núm. 18-19, enero-diciembre de 2003.

Bulygin, Eugenio y MendoncA, Daniel, Normas y sistemas normativos, Madrid, Marcial Pons, 2005.

CARBonell, Miguel (ed.), Neoconstitucionalismo(s), Madrid, Trotta, 2003.

Cueto RÚA, Julio, Judicial Methods of Interpretation of the Law, The Publications Institute Paul M Hebert: Law Center Louisiana State University, 1981, capítulo II.

ESTRADA VÉlEZ, Sergio Iván, Los principios jurídicos y el bloque de constitucionalidad, 2a. ed., Medellín, Universidad de Medellín, 2006.

FOERSTER, Heinz von, Observing Systems: Selected Papers of Heinz von Foerster, Seaside, CA, Intersystems Publications, 1981.

FRANÇOIS, Charles, Diccionario de teoría de sistemas y cibernética, Buenos Aires, Ed. Gesi, 1991.

(ed.), International Encyclopedia of Systemics and Cibernetics, 2a. ed., München, K.G.Knaur, 2004. 
GRÜN, Ernesto, "Los nuevos sistemas jurídicos del mundo globalizado", Revista Telemática de Filosofía del Derecho (Rtfd), núm. 4 00/01, www.filosofiayderecho.com $/$ rtfd/numero4/istemas_ficha.htm.

, "Derecho y caos. Sobre la actual y futura evolución del derecho", Revista Telemática de Filosofía del Derecho (Rtfd), núm. 3- 99/00, www.filosofiayderecho. com/rtfd/numero3/caos.htm.

, "El derecho posmoderno: un sistema lejos del equilibrio", Revista Telemática de Filosofía del Derecho (Rtfd), núm. 1- 97/98, www.filosofiayderecho.com/ rtfd/numerol/posmoderno.html.

, "La aplicación de la sistémica y la cibernética al derecho", Panóptica, 2007, www.panoptica.org/8marcoabrilO7.htm.

, "La crisis del sistema jurídico-político argentino. Un enfoque sistémico y cibernético", Asociación Argentina de Filosofia del Derecho. Conferencias y Ponencias; www.aafd.org.ar/filosofia/documentos/16_Grun\%20 Ernesto.doc.

, "La globalización del derecho: un fenómeno sistémico y cibernético", Revista Telemática de Filosofía del Derecho (Rtfd), núm. 2- 98/99, www. filosofiaydere cho.com/rtfd/numero2/global.html.

, "Una visión sistémica y cibernética del derecho" (libro en versión electrónica), Revista Telemática de Filosofía del Derecho (Rtfd), núm. 3- 99/00, www.filoso fiayderecho.com/rtfd/numero3/grun.htm.

—_, "El derecho después del 11 de septiembre del 2001", Revista Telemática de Filosofía del Derecho (Rtfd), núm. 5, 01/02 www.filosofiayderecho.com/rtfd/nu mero5/11-9-01.htm.

, "El derecho en el mundo globalizado del siglo XXI desde una perspectiva sistémico-cibernética”, Revista Telemática de Filosofía del Derecho, núm. 4, (2000/ 
2001), capítulos I y II, http://www.filosofiay derecho.com/rtfd/numero4/globalizado.htm.

, "La aplicación de la sistémica y la cibernética al derecho", en BOTERO BERNAL, Andrés y ESTRADA VÉLEZ, Sergio Iván (comp.), Temas de filosofía del derecho, Medellín, Señal Editora y Universidad de Medellín, 2003.

, "La teoría general de sistemas y la tributación", Revista La Información, t. LX.

- "Mediación: un enfoque sistémico-cibernético", Transformación de Conflictos en el Mundo Hispánico; http://www.inter-mediacion.com/cibernetico.htm.

—_ "Sistema jurídico y sistema ecológico. Un enfoque sistémico", La Ley - Revista Actualidad, agosto 19 de 1993.

_- "Un enfoque sistémico-cibernético de la mediación", La Ley - Suplemento de métodos alternativos de resolución de conflictos, núm. 2.

cho", Revista La Ley, 1988, A.

__. Una visión sistémica y cibernética del derecho en el mundo globalizado del siglo XXI, Buenos Aires, Dunken, 2004.

—_, Una visión sistémica y cibernética del derecho en el mundo globalizado del siglo XXI, México-Buenos Aires, Lexis Nexis-UNAM, Instituto de Investigaciones Jurídicas, 2006.

_- Una visión sistémica y cibernética del derecho, Buenos Aires, Abeledo Perrot, 1995.

—, "La globalización jurídica", Transformación de conflictos en el Mundo Hispánico, http://www.inter-me diacion.com/grun.htm.

GuIBOURG, Ricardo, Derecho, sistema y realidad, Buenos Aires, Astrea, 1986. 
KuHN, Thomas, La estructura de las revoluciones científicas, trad. Agustín Contin, México, Fondo de Cultura Económica, 1980.

LAMBERT, Edouard, Il governo dei giudici e la lotta contro la legislazione sociale negli Stati Uniti, Milán, Giuffrè, 1996.

LÓPEz, Diego, Teoría impura del derecho: la transformación de la cultura jurídica latinoamericana, Bogotá, Universidad de los Andes, Legis y Universidad Nacional de Colombia, 2004.

LuHMAnn, Niklas, Autopoietc Law: A New Approach to Law and Society, Berlín, Walter de Gruyter, 1988.

NARVÁEZ HERNÁNDEZ, José Ramón, “Arquitectura jurídica: certeza y seguridad en el derecho", Revista Telemática de Filosofía del Derecho, núm. 6, 2002-2003, www.fi losofiayderecho.com.

OlivecronA, Karl, El derecho como hecho, trad. J. J. Santa Piner, Buenos Aires, Depalma, 1959.

Pound, Roscoe, "Causes of Popular Dissatisfaction with the Administration of Justice", American Law Review, 40, septiembre-octubre de 1906.

Romano, Santi, L'ordinamento giuridico, Firenze, Sansoni, 1946.

Ross, Alf, Sobre el derecho y la justicia, trad. Genaro Carrió, Buenos Aires, Eudeba, 1963.

SCHMILL, U., "El modelo del mandato, el orden jurídico y las normas de competencia" (en línea), Cuadernos Electrónicos de Filosofía del Derecho, núm. 5, 2002; http://www.uv.es/CEFD/5/schmill.html.

ZAGREBELSKY, Gustavo, El derecho dúctil: ley, derechos, justicia, Madrid, Trotta, 1999. 